\title{
VII. Equations for the determination of the motion of a disturbed planet by means of M. Hansen's altered time
}

\section{Rev. Brice Bronwin}

To cite this article: Rev. Brice Bronwin (1846) VII. Equations for the determination of the motion of a disturbed planet by means of M. Hansen's altered time, Philosophical Magazine Series 3, 28:184, 20-24, DOI: 10.1080/14786444608645348

To link to this article: http://dx.doi.org/10.1080/14786444608645348

曲 Published online: 30 Apr 2009.

Submit your article to this journal $[\pi$

Џll Article views: 2

Q View related articles $₫$ 
liquid, which was quite clear, gave a much darker tint with the test than any which had previously been obtained in the experiment. It gave a tint about equal to that given with the unfiltered liquid when diluted with its own volume of water; while it (i.e. the last filtered portion) required to be diluted with twice its volume of water to produce the same tint as that given by the reserved filtered portion $(a)$. 'The liquid now passed through the filter very slowly; it was tested again, when eight more fluid ounces had passed through, with the same result as before, except that the tint was a trifle darker.

This experiment sufficiently shows that the effect contemplated does occur, and that it would be unsafe to trust to the action of a filter to separate oxide of lead from water for an unlimited time.

VII. Equations for the Determination of the Motion of a Disturbed Planet by means of M. Hansen's Altered Time. By the Rev. Brice Bronwin*.

THE theory of M. Hansen on Lunar and Planetary Perturbations, owing to the two times $\tau$ and $t$ which it contains, is attended with many difficulties, and is very perplexing. His results I think are in an advantageous form; but perhaps they might be obtained more easily by the equations given in this paper, which are referred to the plane of the orbit as if it were a fixed plane, because I have proved that so referrect they are true. [See this Magazine for November 1844, and also the Cambridge Mathematical Journal, No. 24.]

The equation

$$
\frac{h^{2}}{\mu r}=1+e \cos (u-\pi)=1+e \cos \pi \cos v+e \sin \pi \sin v
$$

is true for the disturbed orbit; $h, e$, and $\pi$ having their known variable values depending on the disturbing force. If $h_{0}, e_{0}$, and $\pi_{0}$ be the values of these quantities when the disturbing force is made to vanish, then

$$
\begin{aligned}
e \cos \pi & =e_{0} \cos \pi_{0}+\int(\cos \pi d e-e \sin \pi d \pi), \\
e \sin \pi & =e_{0} \sin \pi_{0}+\int(\sin \pi d e+e \cos \pi d \pi), \\
h^{2} & =h_{0}^{2}+2 \int h d h .
\end{aligned}
$$

These values substituted in the above equation give

* Communicated by the Author. 


$$
\begin{aligned}
& \frac{h_{0}^{2}}{\mu r}=1+e_{0} \cos \left(v-\pi_{0}\right)-\frac{2}{\mu r} \int h d h \\
& +\cos v \int(\cos \pi d e-e \sin \pi d \pi)+\sin v \int(\sin \pi d e+e \cos \pi d \pi) .
\end{aligned}
$$

Let the constant quantities $\lambda$ and $\rho$ be the same functions of a constant time $\tau$ which $u$ and $r$ are of $t$; then putting the former in place of the latter, we may put them under the sign of integration, changing $\tau$ into $t$ after the integrations are performed. This will change the last equation into

$$
\begin{aligned}
\frac{h_{0}^{2}}{\mu r}= & +e_{0} \cos \left(\nu-\pi_{0}\right)-\frac{2}{\mu_{0}} \int \frac{h d h}{\rho}+\int \cos (\lambda-\pi) d e \\
& +\int e \sin (\lambda-\pi) d \pi .
\end{aligned}
$$

But

$$
\begin{gathered}
\text { But } \quad \int h d h=-\int \frac{d \mathrm{R}}{d v} h d t, \\
d c=-\frac{h d t}{\mu} \sin (v-\pi) \frac{d \mathrm{R}}{d r}-\left(\frac{2 h d t}{\mu r} \cos (v-\pi)+\frac{d r}{\mu} \sin (v-\pi)\right) \frac{d \mathrm{R}}{d v},
\end{gathered}
$$$$
d \pi=\frac{h d t}{\mu e} \cos (v-\pi) \frac{d \mathrm{R}}{d r}-\left(\frac{2 h d t}{\mu r e} \sin (v-\pi)-\frac{d r}{\mu e} \cos (v-\pi)\right) \frac{d \mathrm{R}}{d v} .
$$

The coefficients of $\frac{d \mathbf{R}}{d v}$ are put under the above form for convenience. Substituting these values, we find

$$
\begin{aligned}
\frac{1}{r} & =\frac{\mu}{h_{0}^{2}}+\frac{\mu e_{0}}{h_{0}^{2}} \cos \left(v-\pi_{0}\right)+\frac{1}{h_{0}^{2}} \int \frac{d \mathrm{R}}{d r} \sin (\lambda-v) h d t \\
& +\frac{1}{h_{0}^{2}} \int \frac{d \mathrm{R}}{d v}\left\{\sin (\lambda-v) d r-\frac{2 h d t}{r} \cos (\lambda-v)+\frac{2 h d t}{\rho}\right\} .
\end{aligned}
$$

To abridge, let this be written

$$
\frac{1}{r}=\frac{\mu}{h_{0}{ }^{2}}+\frac{\mu e_{0}}{h_{0}{ }^{2}} \cos \left(\nu-\pi_{0}\right)+\mathrm{P} .
$$

But if $6 t$ be the progression of the apse,

$$
\begin{gathered}
\cos \left(u-\pi_{0}\right)=\cos \left(u-\varepsilon t-\pi_{0}+6 t\right)=\cos \left(u-\varepsilon t-\pi_{0}\right) \\
-6 t \sin \left(v-6 t-\pi_{0}\right),
\end{gathered}
$$

neglecting higher powers of $6 t$. Therefore

$$
\frac{1}{r}=\frac{\mu}{h_{0}{ }^{2}}\left\{1+e_{0} \cos \left(\nu-6 t-\pi_{0}\right)-e_{0} 6 t \sin \left(u-6 t-\pi_{0}\right)\right\}+\mathrm{P} \text {. }
$$

Terms similar to the above, containing $t$ in their coefficients, will arise from the development of $P$; and 6 must be so determined as to drive them out, which will be easily done. We may always neglect terms involving the higher powers of $t$. 
Let $r_{1}$ and $v_{1}$ be the same functions of the new time $\zeta_{1}$ and the constants $h_{1}, e_{1}, \pi_{0}, s_{0}$ which $r$ and $v$ are, when there is no disturbing force, of the time $t$ and the constants $h_{0}, e_{0}, \pi_{0}$, and $\varepsilon_{0}$. Also let $u=v_{1}+6 t$. We shall have

$$
\frac{1}{r_{1}}=\frac{\mu}{h_{1}^{2}}\left\{1+e_{1} \cos \left(\nu_{1}-\pi_{0}\right)\right\} \text {. }
$$

We shall not with $M$. Hansen find the $\log$ of $r$, and therefore shall make $\frac{1}{r}=\frac{1}{r_{1}}+p$. Substituting this value, we shall easily find

$$
\begin{aligned}
p=\mu & \left(\frac{1}{h_{0}^{2}}-\frac{1}{h_{1}^{2}}\right)+\mu\left(\frac{e_{0}}{h_{0}^{2}}-\frac{e_{1}}{h_{1}^{2}}\right) \cos \left(v_{1}-\pi_{0}\right) \\
& -\frac{e_{0} 6 t}{h_{0}^{2}} \sin \left(v_{1}-\pi_{0}\right)+\mathrm{P} .
\end{aligned}
$$

Whence $p$ is of the order of the disturbing force, and it has the advantage of requiring only one integration.

In virtue of the supposed equation

we have

$$
r_{1}^{2} d v_{1}=h_{1} d \zeta, \text { or } \frac{d v_{1}}{d \zeta}=\frac{h_{1}}{r_{1}^{2}}
$$

$$
\frac{d v}{d t}=\frac{d v_{1}}{d t}+\epsilon=\frac{d v_{1}}{d \zeta} \frac{d \zeta}{d t}+6=\frac{h_{1}}{r_{1}^{2}} \frac{d \zeta}{d t}+6 .
$$

This value, substituted in the known equation

$$
\frac{d v}{d t}=\frac{1}{r^{2}}\left(h_{0}-\int \frac{d \mathbf{R}}{d v} d t\right)
$$

gives

$$
\frac{d \zeta}{d t}=\left(1+r_{1} p\right)^{2}\left(\frac{h_{0}}{h_{1}}-\frac{1}{h_{1}} \int \frac{d \mathbf{R}}{d v} d t\right)-\frac{\varepsilon r_{1}^{2}}{h_{1}} .
$$

Of the four quantities $h_{0}, h_{1}, e_{0}, e_{1}$, two are to be found in terns of the others, which will be arbitraries of the theory; and the mode of determining them will be obvious after the development is effected.

Putting $\phi$ for the latitude, $i$ for the inclination, $\vartheta$ and $\theta$ for the longitude of the node on the plane. of the orbit and on the fixed plane, we have

$$
\begin{gathered}
\vartheta=\int \cos i d \theta, \sin \phi=\sin i \sin (u-\vartheta) \\
=\sin i(\cos \vartheta \sin v-\sin \vartheta \cos v), \\
\sin i \cos \vartheta=\sin i_{0} \cos \vartheta_{0}+\int(\cos i \cos \vartheta d i-\sin i \sin \vartheta d \vartheta),
\end{gathered}
$$


$\sin i \sin \vartheta=\sin i_{0} \sin \vartheta_{0}+\int(\cos i \sin \vartheta d i+\sin i \cos \vartheta d \vartheta)$.

Substituting these values, and changing $v$ into $\lambda$, and putting it under the sign of integration, we obtain

$$
\begin{aligned}
\sin \phi & =\sin i_{0} \sin \left(\nu-\vartheta_{0}\right)+\int \sin (\lambda-\vartheta) \cos i d i \\
& -\int \cos (\lambda-\vartheta) \sin i d \vartheta .
\end{aligned}
$$

But $\quad d i=\frac{d t}{h \sin i} \frac{d \mathrm{R}}{d \theta}, \quad d \vartheta=-\frac{\cos i d t}{h \sin i} \frac{d \mathrm{R}}{d i}$.

These values being put in the above, it will become

$$
\begin{aligned}
\sin \phi= & \sin i_{0} \sin \left(u-\vartheta_{0}\right)+\int \frac{\cos i d t}{h \sin i} \frac{d \mathbf{R}}{d \theta} \sin (\lambda-\vartheta) \\
& +\int \frac{\cos i d t}{h} \frac{d \mathbf{R}}{d i} \cos (\lambda-\vartheta) .
\end{aligned}
$$

To abridge, we may write this

$$
\sin \phi=\sin i_{0} \sin \left(u-\vartheta_{0}\right)+Q
$$

or $\sin \phi=\sin i_{0} \sin \left(v+\gamma t-\vartheta_{0}\right)-\gamma t \sin i \cos \left(v+\gamma t-\vartheta_{0}\right)+$ Q.

Here $\gamma t$ is the regression of the node, and $\gamma$ is to be so determined as to take away from $\mathbf{Q}$ the terms having $t$ in their coefficients.

If in the equations

$$
\begin{aligned}
\sin \phi & =\sin i \sin (u-\vartheta), \\
\frac{d \sin \phi}{d t} & =\sin i \cos (u-\vartheta) \frac{d u}{d t},
\end{aligned}
$$

we change $\phi, u, i$, and $\vartheta$ into $\Phi_{0}+\delta \Phi, v_{0}+\delta v, i_{0}+\delta i$, and $\vartheta_{0}+\delta \vartheta, \delta \phi$, \&c. being the parts depending on the disturbing force; and if we expand, taking account of the first power only of $\delta \phi$, \&c., we shall find equations of the form

$$
\begin{aligned}
& \delta i=\mathrm{A} \delta \phi+\mathrm{B} \delta u, \\
& \delta \vartheta=\mathrm{C} \delta \phi+\mathrm{D} \delta u .
\end{aligned}
$$

From these we may correct the values of $i$ and $\vartheta$ or $\theta$ by means of the corrections of $\phi$ and $v$ due to the disturbing force, and in doing so we may take account of higher powers of $\delta \phi, \& c$.

I think the development of the preceding equations would be attended with much less difficulty and perplexity than the development of $M$. Hansen's. I have not noticed the reduction to a fixed plane, but must refer for that to the Number of this Magazine for February 1844, where I have given equations particularly adapted to the lunar theory, and leading to results expressed in terms of the true elliptic longitude. 
I see $\mathrm{Mr}$. Cayley has amended his paper of November 1844 . If he would amend it a little further, it would not be amiss. He has now made $p$ a prime number instead of any odd one : $\theta$ is made of the second instead of the general form. In the expression of $\phi_{1} x$, or rather $\phi x^{\prime}$, he should have had the transformed function $x^{\prime}=\frac{x}{\beta}$, not $x, \beta$ a function of $\theta$. Moreover,

$$
\left.\omega^{\prime}=\frac{1}{p \beta}(\alpha \omega+6 v), \quad v^{\prime}=\frac{1}{p \beta} a^{\prime} \omega+\epsilon^{\prime} v\right) .
$$

Some other amendments are greatly wanted. When $x$ has a determinate value, $x^{\prime}$ should have one also, since $\beta$ is a known function of $\theta$. And if we know what value to assign to $x$, we should have the value of $x^{\prime}$, which is the complete function. I have no room to enlarge, and as Mr. Cayley has done nothing which invalidates what I have done, it is unnecessary.

Gunthwaite Hall, Dec. 12, 1845.

\title{
VIII. On some Points in the Meteorology of Bombay.
} By Lieut.-Colonel SABINE, R.A., F.R.S.*

[With a Plate.]

\begin{abstract}
TN a communication which $I$ had the honour to make to the Section at the York meeting of the British Association, on the subject of the meteorological observations made at Turonto in Canada in the years 1840 to $184.2+$, I noticed some of the advantages which were likely to result to the science of meteorology, from the resolution of the barometric pressure into its two constituents of aqueous and of gaseous pressure. It was shown that when the constituents of the barometric pressure at Toronto were thus disengaged from each other and presented separately, their annual and diurnal variations exhibited a very striking and instructive accordance with the annual and diurnal variations of the temperature. The characteristic features of the several variations when projected in curves were seen to be the same, consisting in all cases of a single progression, having one ascending and one descending branch; the epochs of maxima and minima of the prossures being the same, or very nearly the same, with those of the maxima and minima of temperature; and the correspondence in other respects being such as to manifest the existence of a very intimate connexion between the periodical variations of the temperature, and those of the elastic forces of the air and vapour. The curve of gaseous pressure was inverse in respect to the other two; that is to say, as the temperature increased the clastic force of the vapour increased also, but that of the air diminished, and vice versa ; and this was the case both in the annual and the diurnal variations.

* Communicated to the Mathematical and Physical Section of the British Association for the Adrancement of Science, at the Cambridge Meeting, 1845.

+ See Phil. Mag., vol. xxvi. p. 94.
\end{abstract}

